\title{
A modified perturbation scheme for structural statics systems with random parameters
}

Received: 26 September 2013 / Accepted: 29 January 2014 / Published online: 8 March 2014

(C) The Author(s) 2014. This article is published with open access at Springerlink.com

\begin{abstract}
An answer to the question how random variations of design parameters affect the static structural response is presented in the paper. A variational approach for structural statics is formulated in the context of the finite element method, and stochastic sensitivity of static response is described in terms of the adjoint system technique. When compared with the conventional perturbations, the suggested technique seems to be original, as being completely second-order accurate. Illustrative examples are dealt with beam and shell elements. Numerical results are given for the first two probabilistic moments of displacement sensitivity gradients with respect to random design parameters. Concluding remarks point out to the need for stochastic sensitivity analysis for a better description of real objects, indicating that dynamic stochastic sensitivity analysis as worthy forthcoming work.
\end{abstract}

Keywords Stochastic $\cdot$ Sensitivity $\cdot$ Finite element · Completely second-order accuracy

\section{Introduction}

It is known that the most effective computational tool in civil engineering nowadays is the finite element method (FEM) [1-3], as the basis of almost all structural analysis computer codes. On the other hand, in modern design, sensitivity analysis cannot be avoided, since it makes it possible to determine the so-called starting point (or design point), leading to the optimal solution. Recently, the sensitivity issues are discussed extensively in the literature. Background of the design sensitivity analysis is presented in [4,5], for instance. The sensitivity analysis can be carried out with respect to local design variables, such as cross-sectional area, element thickness, Young's modulus, Poisson's ratio, loading [6-8], or global design variables, e.g., structural member length, overall geometry, overall shape with such more sophisticated mathematic tools as directional and/or topological derivatives $[9,10]$.

In accordance with developments of the computational technique, uncertainties of the design variables appear to be necessarily needed in the state-of-the-art methodologies in computer terms. Besides the traditional Monte Carlo simulation, we may mention the spectral approach [11] and, seeming more effective, perturbational approach [12-15]. In the latter, all the functions of random variables are expanded exponentially. By using the first two probabilistic moments for random variables on input, the first two probabilistic moments of the structural response are obtained on output; the expectations are second-order accurate, while the crosscovariances are first-order accurate $[16,17]$. Formulations for the stochastic sensitivity are followed naturally, $[16,18,19]$, based upon the perturbation coefficients.

A. Jablonka $(\varangle) \cdot$ T. D. Hien

Structure Theory Department, West Pomeranian University of Technology, Al. Piastow 50, 70-311 Szczecin, Poland

E-mail: ajablonka@zut.edu.pl

T. D. Hien

E-mail: tdhien@ps.pl 
In this paper, a modified version of the perturbation schemes presented so far in the literature is proposed for linear elastic structural systems. With the same input data as for the conventional perturbational techniques, in the suggested scheme, both the two output moments are with second-order accuracy. This is the goal of the paper.

After the introduction, in the second section, a variational basis of FEM is given. Starting from the Hamilton's principle, the total energy balance is formulated, and the equilibrium equations are obtained in the context of FEM. In the next section, the stochastic static sensitivity is described. In the fourth section, analysis results are presented for three structural systems, modeled with different types of finite elements-truss, beam and shell ones, suggesting remarks finish the paper.

\section{Stochastic finite element model of statics}

It is known that the variational Hamilton's principle states that among all permissible displacement fields, satisfying boundary and initial conditions at times $t_{1}, t_{2}$ in the volume $\Omega$, the real field makes stationary the functional of the total energy. In statics, the total energy is the sum of potential energy $V$ and external forces work $W$, leading to a specific form-the minimum potential principle. That is

$$
\delta(V-W)=0
$$

with

$$
\begin{aligned}
& V=\int_{\Omega} \frac{1}{2} \boldsymbol{\varepsilon}^{\mathrm{T}} \boldsymbol{\sigma} \mathrm{d} \Omega \\
& W=\int_{\partial \Omega} \widehat{\mathbf{t}}^{\mathrm{T}} \mathbf{u} \mathrm{d}(\partial \Omega)
\end{aligned}
$$

where $\mathbf{u}=\left\{u_{i}\right\}, \boldsymbol{\varepsilon}=\left\{\varepsilon_{11}, \varepsilon_{22}, \varepsilon_{33}, \sqrt{2} \varepsilon_{12}, \sqrt{2} \varepsilon_{13}, \sqrt{2} \varepsilon_{23}\right\}, \boldsymbol{\sigma}=\left\{\sigma_{11}, \sigma_{22}, \sigma_{22}, \sqrt{2} \sigma_{12}, \sqrt{2} \sigma_{13}, \sqrt{2} \sigma_{23}\right\}$ and $\widehat{\mathbf{t}}=\left\{\widehat{t_{i}}\right\}, i=1,2,3$, are the displacement, strain, stress and the boundary force vectors, respectively. By the engineering character of the paper, in Eq. (3), the body forces are neglected.

Implementing the finite elements setting, the FEM equilibrium equation system is obtained as

$$
\mathbf{K} \mathbf{q}=\mathbf{Q}
$$

where $\mathbf{K}=\left[K_{\alpha \beta}\right], \mathbf{q}=\left\{q_{\alpha}\right\}$ and $\mathbf{Q}=\left\{Q_{\alpha}\right\}, \alpha=1,2, \ldots, N$, are the system stiffness matrix, nodal displacement and load vectors, respectively, with $N$ being the system number of degrees of freedom. The explicit forms of the stiffness and nodal load matrices for specific types of the finite elements can be found in [1-3], for instance.

Equation (4) may be rewritten in the residual form, where twice repeated indices implying summation as

$$
Q_{\alpha}-K_{\alpha \beta} q_{\beta}=0
$$

and this indicial notation will be used from now on.

\section{Stochastic sensitivity of static response}

The structural response of a multi-degree-of-freedom system can be defined by the function

$$
\Phi=\Phi\left[q_{\alpha}(\mathbf{h}, \mathbf{b}), \mathbf{h}\right]
$$

satisfying the equilibrium condition (4) and being an explicit and implicit function of the vector of design variables $\mathbf{h}=\left\{h^{d}\right\}, d=1,2, \ldots, D$, and the vector of random variables $\mathbf{b}=\left\{b^{r}\right\}, r=1,2, \ldots, R$, i.e.,

$$
Q_{\alpha}(\mathbf{h}, \mathbf{b})-K_{\alpha \beta}(\mathbf{h}, \mathbf{b}) q_{\beta}(\mathbf{h}, \mathbf{b})=0, \quad \alpha, \beta=1,2, \ldots, N
$$

The random variables $b^{r}$ can be defined via the first two probabilistic characteristics-expectations $\bar{b}^{r}$ and cross-covariances $\operatorname{Cov}\left(b^{r}, b^{s}\right)$ as 


$$
\begin{aligned}
\bar{b}^{r} & =\mathrm{E}\left[b^{r}\right]=\int_{-\infty}^{+\infty} b^{r} p\left(b^{r}\right) \mathrm{d} b^{r} \\
\operatorname{Cov}\left(b^{r}, b^{s}\right) & =\mathrm{E}\left[\left(b^{r}-b_{0}^{r}\right)\left(b^{s}-b_{0}^{s}\right)\right]=\mathrm{R}\left(b^{r}, b^{s}\right) \sqrt{\operatorname{Var}\left(b^{r}\right) \operatorname{Var}\left(b^{s}\right)}
\end{aligned}
$$

with

$$
\begin{aligned}
\mathrm{R}\left(b^{r}, b^{s}\right) & =\int_{-\infty}^{+\infty} \int_{-\infty}^{+\infty} b^{r} b^{s} p\left(b^{r}, b^{s}\right) \mathrm{d} b^{r} \mathrm{~d} b^{s} \\
\operatorname{Var} & =\alpha^{2} \mathrm{E}^{2}\left[b^{r}\right]
\end{aligned}
$$

where $\mathrm{R}\left(b^{r}, b^{s}\right), \operatorname{Var}\left(b^{r}, b^{s}\right), p\left(b^{r}, b^{s}\right)$ and $\alpha$ denote functions of correlation, variance, joint probability density and the coefficient of variation, respectively.

The functions of random variables $q_{\alpha}, K_{\alpha \beta}$ and $Q_{\alpha}$ can be handled with the finite difference technique or by the leas square fit method, cf. [20], for instance. In this paper, a perturbation scheme will be employed. Suppose that $K_{\alpha \beta}, q_{\beta}$ and $Q_{\alpha}$ are twice differentiable with respect to $h^{d}$. Using the chain rule of differentiation leads to

$$
\Phi^{; d}=\Phi^{d d}+\Phi_{. \alpha} q_{\alpha}^{; d}
$$

where $(.)^{; d}$ is the first ordinary derivative with respect to the $d$ th design variable, while $(\cdot)^{d}$ and $(\cdot)_{. \alpha}$ are the first partial derivatives with respect to the $d$ th design variable and $\alpha$ th nodal displacement, respectively. Because $\Phi$ is an explicit function of its arguments, the components $\Phi^{d}$ and $\Phi_{. \alpha}$ are known. The derivatives $q_{\alpha}^{; d}$ must be determined, since $q_{\alpha}$ are implicit with respect to $h_{d}$. Differentiating the equilibrium Eq. (7) with respect to $h_{d}$ yields

$$
K_{\alpha \beta} q_{\beta}^{; d}=Q_{\alpha}^{. d}-K_{\alpha \beta}^{. d} q_{\beta}
$$

To eliminate $q_{\alpha}^{; d}$ from (12), the adjoint system method is used. The adjoint vector $\lambda_{\alpha}$ may be defined so that the adjoint equations system takes the form

$$
K_{\alpha \beta} \lambda_{\beta}=\Phi_{. \alpha}
$$

that, substituted (14) into (12) and on account of (13), implies

$$
\Phi^{; d}=\Phi^{. d}+\lambda_{\alpha}\left(Q_{\alpha}^{. d}-K_{\alpha \beta}^{. d} q_{\beta}\right)
$$

As mentioned above, the functions of random variables $K_{\alpha \beta}, Q_{\alpha}, \Phi_{, \alpha}, q_{\beta}, \lambda_{\alpha}, K_{\alpha \beta}^{. d}, Q_{\alpha}^{. d}$ and $\Phi^{. d}$ are now expanded around the expectations $\bar{b}^{r}$ via the second-order perturbation, with a given small parameter $\theta$, symbolically written as

$$
(\cdot)(\mathbf{h}, \mathbf{b})=(\cdot)^{0}+\theta(\cdot)^{; r} \Delta b^{r}+\frac{1}{2} \theta^{2}(\cdot)^{; r s} \Delta b^{r} \Delta b^{s}, \quad r, s=1,2, \ldots, R
$$

in which $\Delta b^{r}$ denotes the perturbational increment of $b^{r}$ with respect to $b_{0}^{r}$, and (..) ${ }^{0}(\cdot)^{; r}$ and (.); ${ }^{; r s}$ describe the zeroth, first and mixed (second) ordinary derivatives with respect to $b^{r}$.

Substituting the expansions of $K_{\alpha \beta}, Q_{\alpha}, \Phi_{. \alpha}, q_{\beta}$ and $\lambda_{\alpha}$ into (7) and (14) and equating the coefficients of the parameter $\theta$ to zeroth, first and second power, we obtain

- 1 pair of the zero-order equations

$$
\begin{aligned}
& K_{\alpha \beta}^{0} q_{\beta}^{0}=Q_{\alpha}^{0} \\
& K_{\alpha \beta}^{0} \lambda_{\beta}^{0}=\Phi_{. \alpha}^{0}
\end{aligned}
$$

- $\quad r$ pairs of the first-order equations

$$
\begin{aligned}
& K_{\alpha \beta}^{0} q_{\beta}^{; r}=Q_{\alpha}^{; r}-K_{\alpha \beta}^{; r} q_{\beta}^{0} \\
& K_{\alpha \beta}^{0} \lambda_{\beta}^{; r}=\Phi_{. \alpha}^{; r}-K_{\alpha \beta}^{; r} \lambda_{\beta}^{0}, \quad r, s=1,2, \ldots, R
\end{aligned}
$$


- 1 pair of the second-order equations

$$
\begin{aligned}
& K_{\alpha \beta}^{0} q_{\beta}^{(2)}=\left(\frac{1}{2} Q_{\alpha}^{; r s}-K_{\alpha \beta}^{; r} q_{\beta}^{, s}-\frac{1}{2} K_{\alpha \beta}^{; r s} q_{\beta}^{0}\right) \operatorname{Cov}\left(b^{r}, b^{s}\right) \\
& K_{\alpha \beta}^{0} \lambda_{\beta}^{(2)}=\left(\frac{1}{2} \Phi_{. \alpha}^{; r s}-K_{\alpha \beta}^{; r} \lambda_{\beta}^{, s}-\frac{1}{2} K_{\alpha \beta}^{; r s} \lambda_{\beta}^{0}\right) \operatorname{Cov}\left(b^{r}, b^{s}\right)
\end{aligned}
$$

where

$$
\begin{aligned}
& q_{\alpha}^{(2)}=\frac{1}{2} q_{\alpha}^{; r s} \operatorname{Cov}\left(b^{r}, b^{s}\right) \\
& \lambda_{\alpha}^{(2)}=\frac{1}{2} \lambda_{\alpha}^{; r s} \operatorname{Cov}\left(b^{r}, b^{s}\right)
\end{aligned}, \quad r, s=1,2, \ldots, R
$$

Having solved for $q_{\beta}^{0}, q_{\beta}^{; r}$ and $q_{\beta}^{(2)}$ in (17) $1,(18)_{1}$ and (19) $)_{1}$, the first two probabilistic moments for $q_{\alpha}$ can be computed by using the expansion (16) with $\theta=1$, i.e.,

$$
q_{\alpha}(\mathbf{h}, \mathbf{b})=q_{\alpha}^{0}+q_{\alpha}^{; r} \Delta b^{r}+\frac{1}{2} q_{\alpha}^{; r s} \Delta b^{r} \Delta b^{s}, \quad r, s=1,2, \ldots, R
$$

The expectation vector for $q_{\alpha}$ can then be obtained as

$$
\bar{q}_{\alpha}=q_{\alpha}^{0}+q_{\alpha}^{(2)}
$$

To compute the cross-covariance matrix, we note that, cf. (21) and (22),

$$
\Delta q_{\alpha}=q_{\alpha}-\mathrm{E}\left[q_{\alpha}\right]=q_{\alpha}^{; r} \Delta b^{r}+\frac{1}{2} q_{\alpha}^{; r s} \Delta b^{r} \Delta b^{s}-q_{\alpha}^{(2)}
$$

with $q_{\alpha}^{(2)}$ being a deterministic quantity so that $\operatorname{Cov}\left(q_{\alpha}, q_{\beta}\right)=\mathrm{E}\left[\Delta q_{\alpha} \Delta q_{\beta}\right]$ can be expressed as

$$
\operatorname{Cov}\left(q_{\alpha}, q_{\beta}\right)=q_{\alpha}^{; r} q_{\beta}^{; s} \operatorname{Cov}\left(b^{r}, b^{s}\right)-q_{\alpha}^{(2)} q_{\beta}^{(2)}
$$

It should be pointed out here that both the solutions (22) and (24) are second-order accurate, when compared with the 'conventional' ones, $[12,13,16]$, in which only the expectation vector is second-order accurate, while the cross-covariance matrix is first-order accurate.

When $q_{\alpha}^{0}$ and $\lambda_{\alpha}^{0}$ from (17) are known, the functions $q_{\alpha}^{; r}, \lambda_{\alpha}^{; r}, q_{\alpha}^{(2)}$ and $\lambda_{\alpha}^{(2)}$ can be solved by (18) and (19) in a sequential manner. In this way, it is possible to calculate the probability distribution of sensitivity. The expectations and cross-covariances of the sensitivity gradient are then written as, cf. [17]

$$
\begin{aligned}
\mathrm{E}\left[\Phi^{d}\right]= & G^{0 . d}+\frac{1}{2} G^{. d ; r s} \operatorname{Cov}\left(b^{r}, b^{s}\right)+\mathcal{A}_{\alpha}^{d}\left(\lambda_{\alpha}^{0}+\lambda_{\alpha}^{(2)}\right) \\
& -k_{\alpha \beta}^{0 . d} q_{\beta}^{(2)} \lambda_{\alpha}^{0}+\left(\mathcal{B}_{\alpha}^{d r} \lambda_{\alpha}^{; s}+\mathcal{C}_{\alpha}^{d r s} \lambda_{\alpha}^{0}\right) \operatorname{Cov}\left(b^{r}, b^{s}\right)
\end{aligned}
$$

and

$$
\begin{aligned}
\operatorname{Cov}\left(\Phi^{. d}, \Phi^{. e}\right)= & {\left[G^{. d ; r} G^{. e ; s}+\left(G^{. d ; r} \mathcal{A}_{\alpha}^{e}+G^{. e ; r} \mathcal{A}_{\alpha}^{d}\right) \lambda_{\alpha}^{; s}\right.} \\
& +\left(G^{. d ; r} \mathcal{B}_{\alpha}^{e s}+G^{. e ; r} \mathcal{B}_{\alpha}^{d s}\right) \lambda_{\alpha}^{0} \\
& +\mathcal{A}_{\alpha}^{d} \mathcal{A}_{\beta}^{e} \lambda_{\alpha}^{; r} \lambda_{\beta}^{; s}+\left(\mathcal{A}_{\alpha}^{d} \mathcal{B}_{\beta}^{e r}+\mathcal{A}_{\beta}^{e} \mathcal{B}_{\alpha}^{d r}\right) \\
& \left.\times \lambda_{\alpha}^{; s} \lambda_{\beta}^{0}+\mathcal{B}_{\alpha}^{d r} \mathcal{B}_{\beta}^{e s} \lambda_{\alpha}^{0} \lambda_{\beta}^{0}\right] \operatorname{Cov}\left(b^{r}, b^{s}\right) \\
& -\left[\bar{\Phi}^{. d}\left(\frac{1}{2} G^{. e ; r s}+\mathcal{B}_{\alpha}^{e r} \lambda_{\alpha}^{; s}+\mathcal{C}_{\alpha}^{e r s} \lambda_{\alpha}^{0}\right)\right. \\
& \left.+\bar{\Phi}^{e}\left(\frac{1}{2} G^{. d ; r s}+\mathcal{B}_{\alpha}^{d r} \lambda_{\alpha}^{; s}+\mathcal{C}_{\alpha}^{d r s} \lambda_{\alpha}^{0}\right)\right] \operatorname{Cov}\left(b^{r}, b^{s}\right) \\
& -\left[\bar{\Phi}^{\cdot d}\left(\mathcal{A}_{\alpha}^{e} \lambda_{\alpha}^{(2)}-k_{\alpha \beta}^{0 . e} q_{\beta}^{(2)} \lambda_{\alpha}^{0}\right)\right. \\
& \left.+\bar{\Phi}^{\cdot e}\left(\mathcal{A}_{\alpha}^{d} \lambda_{\alpha}^{(2)}-k_{\alpha \beta}^{0 . d} q_{\beta}^{(2)} \lambda_{\alpha}^{0}\right)\right]
\end{aligned}
$$




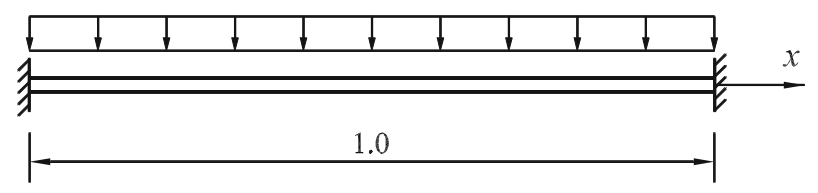

Fig. 1 Clamped-clamped beam

where

$$
\begin{aligned}
\mathcal{A}_{\alpha}^{d} & =Q_{\alpha}^{0 . d}-K_{\alpha \beta}^{0 . d} q_{\beta}^{0} \\
\mathcal{B}_{\alpha}^{d r} & =Q_{\alpha}^{. d ; r}-K_{\alpha \beta}^{0 . d} q_{\beta}^{; r}-K_{\alpha \beta}^{. d ; r} q_{\beta}^{0} \\
\mathcal{C}_{\alpha}^{d r s} & =\frac{1}{2} Q_{\alpha}^{. d ; r s}-K_{\alpha \beta}^{. d ; r} q_{\beta}^{; s}-\frac{1}{2} K_{\alpha \beta}^{. d ; r s} q_{\beta}^{0}
\end{aligned}
$$

with $d, e=1,2, \ldots, D ; r, s=1,2, \ldots, R ; \alpha, \beta=1,2, \ldots, N$. Also, the cross-covariance matrix (26) is obtained with the second-order accuracy, and not the first-order one as in $[16,17]$.

\section{Numerical results}

Numerical illustrations discussed below are first concerned with the well-known examples considered already in [17]. The significant difference, however, is that the numerical results are given as completely second-order accurate for both the expectation vector and cross-covariance matrix. Analysis results are also presented for a real-scale complex structure described in [21,22].

The static sensitivity of displacement to random change of design parameters for two different structural systems is analyzed. Two groups of random variables, geometrical and material parameters, are considered. They are cross-sectional areas of the truss or beam elements, thickness of the plate or shell elements, and Young's modulus of all the elements. The stochastic analysis are carried on by using a modified version of the computer code POLSAP [23].

In Example 1, the displacement and slope responses of the clamped-clamped beam are considered, Fig. 1. The beam is of length $L=10 \mathrm{~m}$ and constant cross-sectional area $A=0.005 \mathrm{~m}^{2}$ and is subjected to a uniformly distributed load $q=5 \mathrm{kN} / \mathrm{m}$. The moments of inertia $I_{y}=I_{z}=A^{2} / 6, I_{x}=I_{y}+I_{z}$ are adopted. The material is characterized by Poisson's ratio $v=0.3$ and Young's modulus $e^{r}, r=1,2, \ldots, 100$, which are the random design variables with the means $e_{0}^{r}=\mathrm{E}\left[e^{r}\right]=0.2 \mathrm{GPa}$. The correlation function is described as

$$
\mathrm{R}\left(e^{r}, e^{s}\right)=\exp \left[-\operatorname{abs}\left(x^{r}-x^{s}\right) / \lambda\right]
$$

$\lambda=0.1$ and the coefficient of variation $\alpha=0.07$.

The response function is defined as

$$
\Phi=\int_{0}^{1} \delta\left(x-x^{*}\right) y(x) \mathrm{d} x
$$

where $\delta(x)$ is the Dirac delta that equals zero to $x \neq 0$, in this case $x=x^{*}$, with $x^{*}$ being a given $x$-ordinate. We assume that both the allowable displacement and slope at the mid-point of the beam are 0.02 .

It is shown from the obtained results that the maximum displacement of the beam does not exceed $1.56 \mathrm{~cm}$, and extremal value of slope is close $5 \times 10^{-2}$. The obtained expectations differ from the deterministic values about $1 \%$, cf. [17].

The numerical results of the displacement sensitivity at the mid-point of the beam to the change of the Young's modulus value are shown in Fig. 2. Clearly, the most sensitive results are received with elements being located at the clamps and at the mid-point of the beam, and the smallest sensitive response is at places where the bending moment is zero, $x \cong 0.22 \mathrm{~L}$ and $x \cong 0.78 \mathrm{~L}$. The values of the standard deviations are about $15-25 \%$ of the expectations for the given parameters. The differences between the expected and deterministic sensitivities are about $3 \%$, not as small as those of the displacements. The differences between the second-order accurate variances obtained here and the first-order accurate ones in [17] are about $1 \%$. 


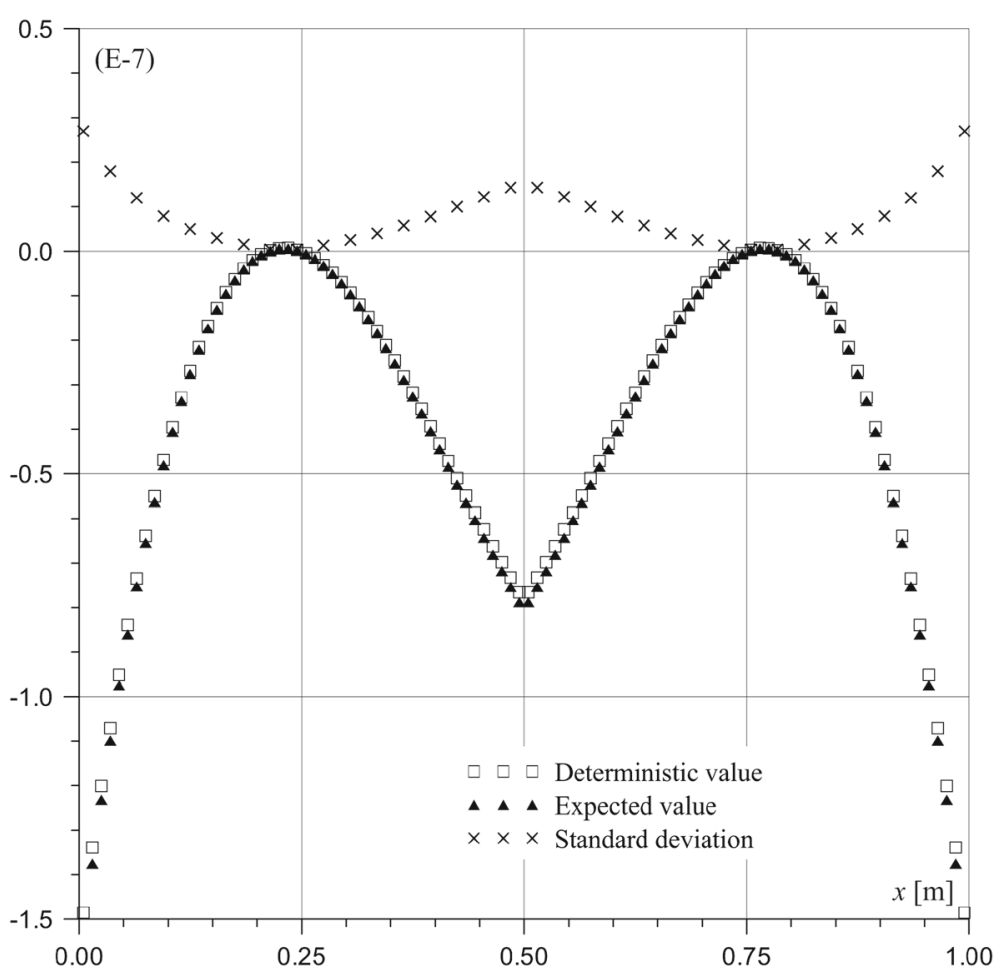

Fig. 2 Displacement sensitivity-Young's moduli as random design variables

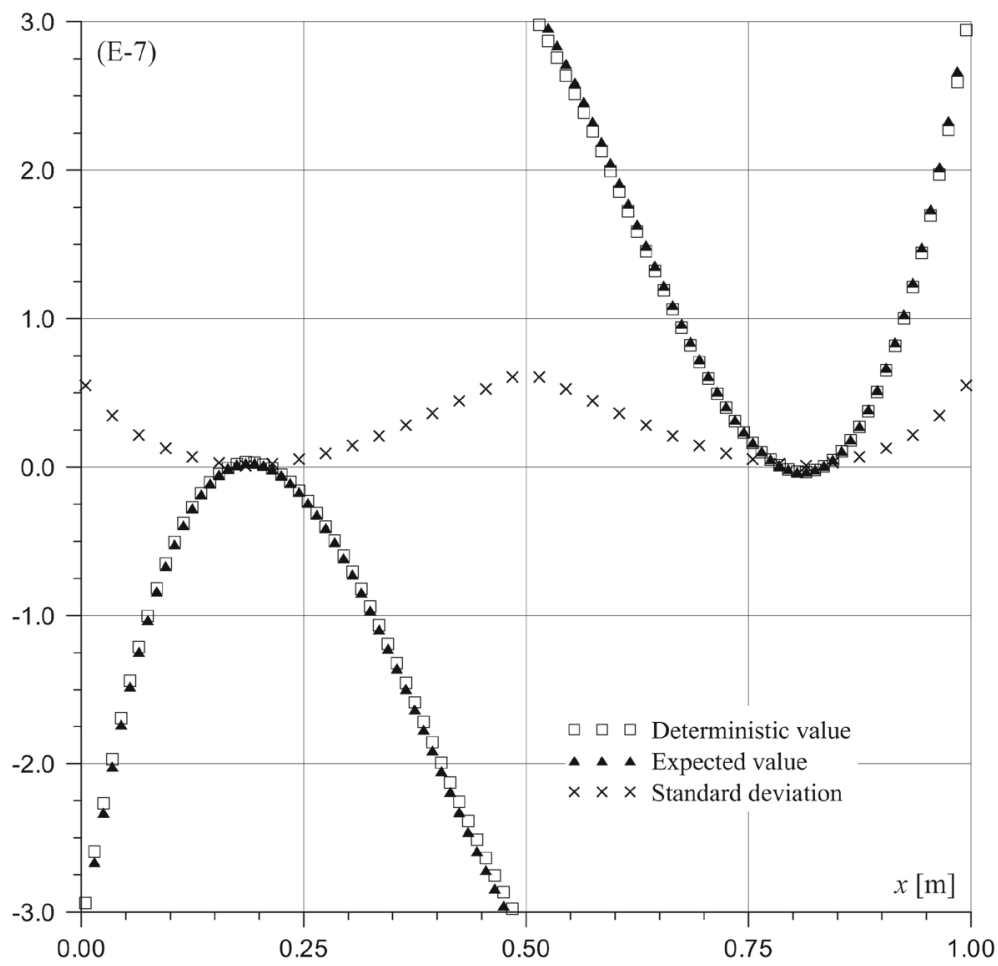

Fig. 3 Slope sensitivity - Young's moduli as random design variables

In the analysis of the slope sensitivity to the change of the Young's modulus value, Fig. 3, the most sensitive elements are located, like in the previous case, at the mid-point of the beam and at the clamps. The elements almost insensitive when $x \cong 0.2 \mathrm{~L}$ and $x \cong 0.8 \mathrm{~L}$ should be caused by round-off computer errors and the FEM- 

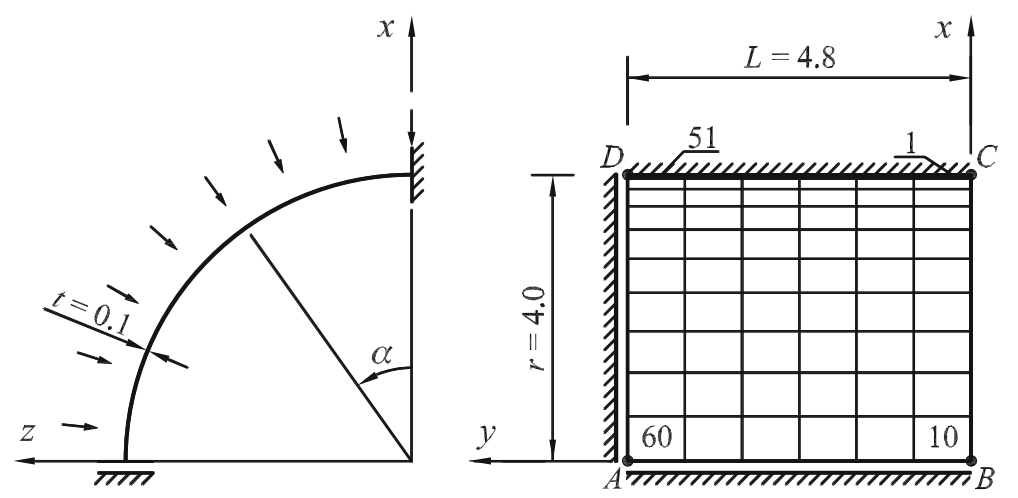

Fig. 4 Cylindrical shell-FEM model

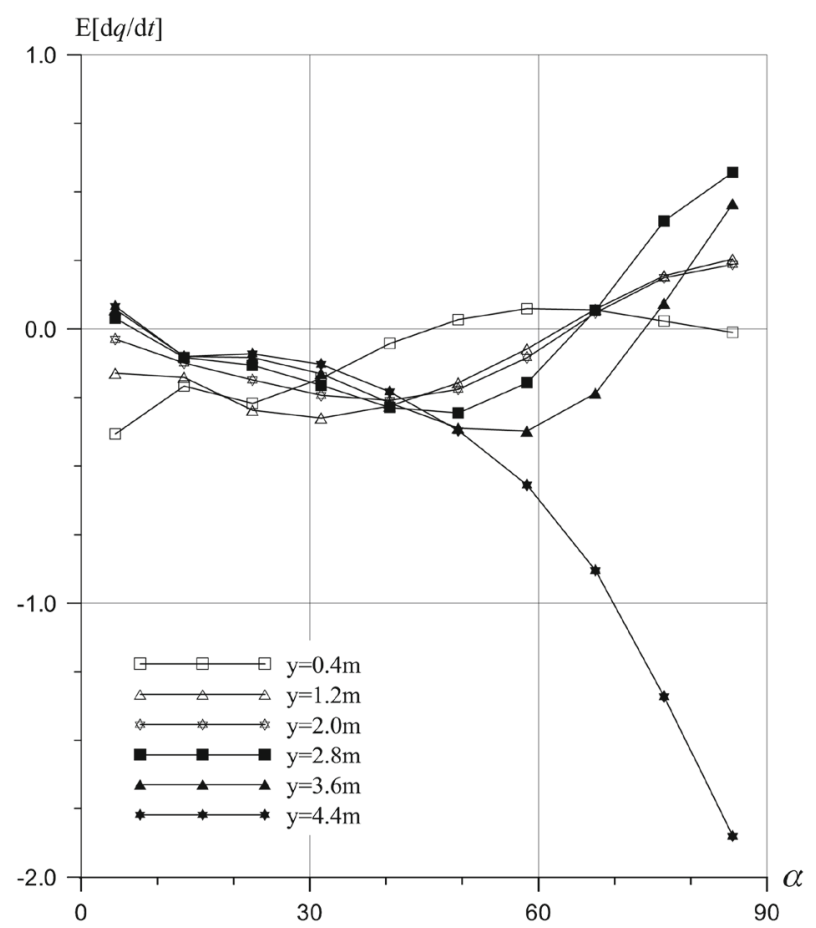

Fig. 5 Expectations of displacement sensitivity—shell thicknesses as random design variables

based inaccuracy. The standard deviation is about $17-25 \%$ of the expectations, and the differences between the excepted and deterministic values are about $3 \%$.

Example 2 concerns the displacement response of a thin shell structure, which is a quarter of a cylinder of radius $r=4.0 \mathrm{~m}$ and length $L=4.8 \mathrm{~m}$, Fig. 4 . The boundary conditions are adopted as follows:: along the bound $A B$, the $x$-displacements and $y$ - and $z$-rotations are zero; $B C$ is entirely free; $C D$ is fixed; along the bound $D A$, the $y$-displacements and $x$ - and $z$-rotations are zero. The material is characterized by Young's modulus $e=10 \mathrm{GPa}$ and Poisson's ratio $v=0.3$. The response function takes also the form (29). The admissible $z$-displacement at the node $\mathrm{A}$ is $0.01 \mathrm{~m}$. The random design variables are defined as the shell thicknesses $t^{r}$, with the expectations $t_{0}^{r}=\mathrm{E}\left[t^{r}\right]=0.1 \mathrm{~m}$. The correlation function is given as

$$
\mathrm{R}\left(t^{r}, t^{s}\right)=\Theta \exp \left\{-\operatorname{abs}\left[\left(x^{r}-x^{s}\right)\left(y^{r}-y^{s}\right)\right] / \lambda\right\}
$$

where $\Theta=1.5 /(\mathrm{rL}), \lambda=2.5 r^{2} L^{2}$ and the coefficient of variation $\alpha=0.1$. The finite element mesh of the shell incudes 60 equally rectangular elements.

Figure 5 shows the expectations of displacement sensitivity of the node $A$ to the change of element thicknesses. It is known that the most sensitive results are obtained with the shell elements being around node $A$. In 


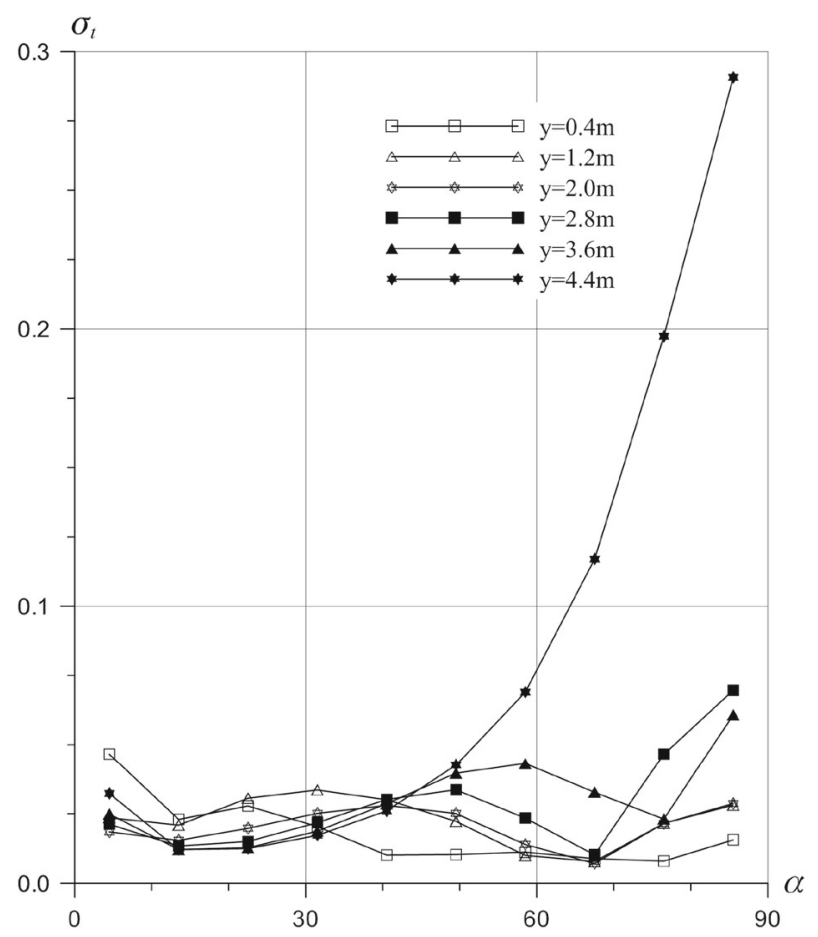

Fig. 6 Standard deviations of displacement sensitivity—shell thicknesses as random design variables

contrast to the beam previously considered, the curves of shell sensitivities are characterized by high irregularity. We observe that some sensitivity values obtained here are positive. In general cases, in the static response of simple structures, the more massive system is, the lesser displacement is obtained. In this case, the displacement response is more complicated at places of the lower part, resulting in those nonnegative values of sensitivity. This means that to decrease the displacement at the considered point $A$, decreasing thickness of shell's elements in appropriate domains should be required in an alternative design point. The standard deviations of displacement sensitivity with respect to the shell thicknesses is about $10-30 \%$ of the expectations, Fig. 6. Comparing the stochastic and deterministic results, it can be concluded that they differ by no more than $3 \%$.

The numerical results of sensitivity analysis of the beam, Figs. 2, 3, and of the shell, Figs. 5, 6, are similar to those presented in [17] at first glance. However, we note some differences, especially in the results of the displacement sensitivity analysis of the shell, Fig. 5. Significant differences are shown in the expectations of displacement sensitivity on the unclamped edge of the shell.

In Example 3, displacement response of a complex structure is considered. The structure is modeled by a FEM mesh with 216 different elements. Three element types are employed-36 truss-, 80 beam- and 100 shell-elements, Fig. 7, resulting in 990 degrees of freedom. As for boundary conditions, nodes 1-4 located at the base of the structure are fixed. The truss and beam elements are designed with steel profiles, characterized by Young's modulus $e=210 \mathrm{GPa}$ and Poisson's ratio $v=0.3$. The random design variables are defined as the cross-sectional areas $a^{r}$, with the expectations $a_{0}^{r}=\mathrm{E}\left[a^{r}\right]=4.324 \times 10^{-3} \mathrm{~m}^{2}$. Clearly, for the sake of simplicity in the sensitivity terms, moments of inertia of the truss and beam elements are considered as a measure of the cross-sectional areas squared, with $I_{y}=I_{z}=0.7552 a^{2}$ and $I_{x}=1.2644 a^{2}$. The shell elements are designed with concrete, which is characterized by Young's modulus $e=30 \mathrm{GPa}$ and Poisson's ratio $v=0.2$. The random design variables are defined as the shell thicknesses $a^{r}$, with the expectations $a_{0}^{r}=\mathrm{E}\left[a^{r}\right]=0.1 \mathrm{~m}$. The correlation function is described as

$$
\mathrm{R}\left(a^{r}, a^{s}\right)=\exp \left\{-\operatorname{abs}\left[\left(x^{r}-x^{s}\right)\left(y^{r}-y^{s}\right)\left(z^{r}-z^{s}\right)\right] / \lambda\right\}
$$

where $\lambda=0.05$ and the coefficient of variation $\alpha=0.04$ are adopted. As before, the response function takes the form (29).

The system is uniformly vertically loaded on the plate, with $q=10 \mathrm{kN} / \mathrm{m}^{2}$. The allowable displacements are assumed as: $u_{x}=0.01$ and $u_{z}=0.03$, separately for each of the two cases. The respective two displacement 


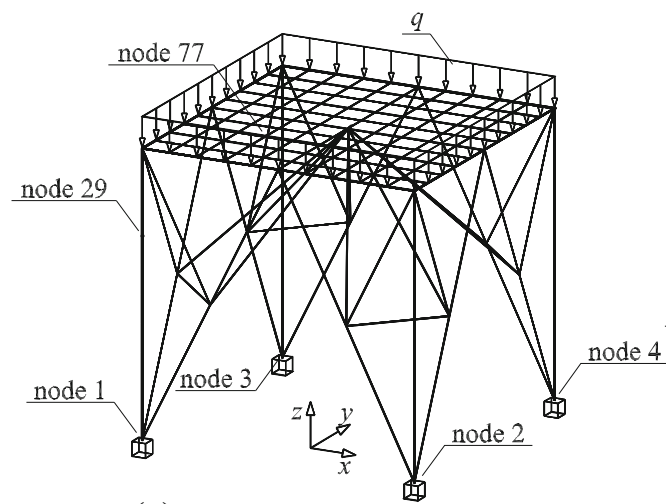

(a)

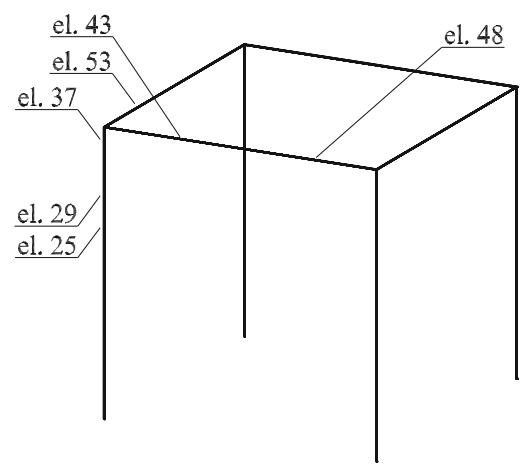

(c)

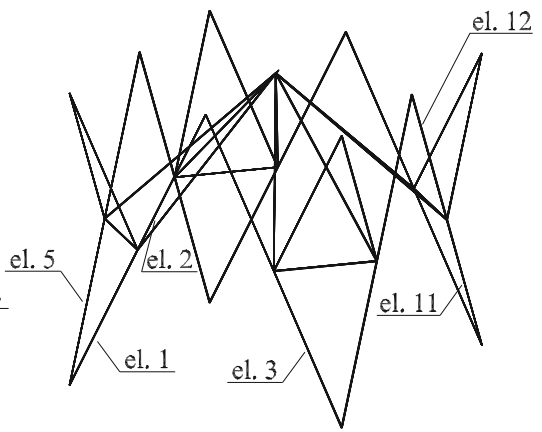

(b)

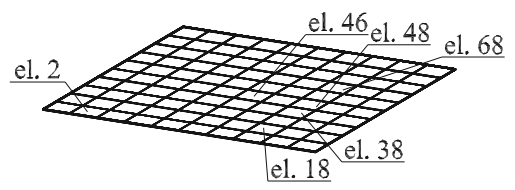

(d)

Fig. 7 Complex structure: a truss-beam-shell FEM model and its $\mathbf{b}$ truss-, $\mathbf{c}$ beam- and $\mathbf{d}$ shell-elements

Table 1 Displacement sensitivity—cross-sectional areas of truss elements as a random design variables

\begin{tabular}{|c|c|c|c|c|c|c|}
\hline \multirow[t]{2}{*}{ El. no. } & \multicolumn{3}{|l|}{ Constr. (1) } & \multicolumn{3}{|l|}{ Constr. (2) } \\
\hline & Deterministic value & Expected value & SD & Deterministic value & Expected value & SD \\
\hline 1 & -17.92494 & -18.37723 & 3.23539 & -2.25746 & -2.31200 & 0.39954 \\
\hline 2 & -7.07349 & -7.25539 & 1.31947 & -0.39619 & -0.40644 & 0.07629 \\
\hline 3 & 10.83438 & 11.10148 & 1.94797 & -2.20266 & -2.25445 & 0.38236 \\
\hline 5 & 3.61791 & 3.73139 & 0.78627 & -2.25746 & -2.31200 & 0.39954 \\
\hline 11 & 6.37701 & 6.51905 & 1.10961 & -1.18967 & -1.21743 & 0.20666 \\
\hline 12 & 2.28362 & 2.33703 & 0.41083 & 0.10139 & 0.10295 & 0.02030 \\
\hline
\end{tabular}

Table 2 Displacement sensitivity—cross-sectional areas of beam elements as a random design variables

\begin{tabular}{|c|c|c|c|c|c|c|}
\hline \multirow[t]{2}{*}{ El. no. } & \multicolumn{3}{|l|}{ Constr. (1) } & \multicolumn{3}{|l|}{ Constr. (2) } \\
\hline & Deterministic value & Expected value & SD & Deterministic value & Expected value & SD \\
\hline 25 & -53.19560 & -56.09343 & 13.60375 & -0.74295 & -0.75953 & 0.11421 \\
\hline 29 & -49.42492 & -51.987880 & 12.31199 & -1.27611 & -1.30509 & 0.19950 \\
\hline 37 & 95.89997 & 102.17179 & 27.44810 & -2.77865 & -2.84255 & 0.43877 \\
\hline 43 & -28.32681 & -28.97225 & 4.81574 & -5.01730 & -5.12153 & 0.78468 \\
\hline 48 & 5.57682 & 5.69576 & 0.91009 & 0.89267 & 0.91146 & 0.16085 \\
\hline 53 & 4.15635 & 4.13444 & 0.17614 & -5.01730 & -5.12153 & 0.78468 \\
\hline
\end{tabular}

constraints are specified as: constr. (1) $-u_{x}$ at node $29(-5.0,-5.0,7.0)$, constr. (2) $-u_{z}$ at node $77(-2.0$, $-2.0,10.0)$. Static sensitivity with respect to the changes, the selected elements are presented in Tables 1,2 and 3.

It is seen that the most sensitive results for the two displacement constrains are obtained with the truss element No. 1, Table 1. Some truss elements, for example, Nos. 3, 5, 11 and 12, are characterized by positive 
Table 3 Displacement sensitivity — thicknesses of shell elements as a random design variables

\begin{tabular}{|c|c|c|c|c|c|c|}
\hline \multirow[t]{2}{*}{ El. no. } & \multicolumn{3}{|l|}{ Constr. (1) } & \multicolumn{3}{|l|}{ Constr. (2) } \\
\hline & Deterministic value & Expected value & SD & Deterministic value & Expected value & SD \\
\hline 2 & -2.31283 & -2.36126 & 0.36072 & -0.23886 & -0.24177 & 0.02060 \\
\hline 18 & 0.20451 & 0.20675 & 0.02094 & -0.02050 & -0.02005 & 0.00297 \\
\hline 38 & 0.19709 & 0.19845 & 0.01538 & 0.08189 & 0.08229 & 0.00552 \\
\hline 46 & -0.61009 & -0.61401 & 0.04329 & -1.28169 & -1.28377 & 0.05302 \\
\hline 48 & 0.18716 & 0.18854 & 0.01471 & 0.09070 & 0.09108 & 0.00533 \\
\hline 68 & 0.08461 & 0.08510 & 0.00574 & 0.09417 & 0.09434 & 0.00403 \\
\hline
\end{tabular}

sensitivity values. This means that increasing their cross-sectional areas enlarges the displacement at the considered nodes. The standard deviations of displacement sensitivity with respect to the truss cross-sectional areas is about $15-25 \%$ of the expectations. Stochastic and deterministic results differ by about $3 \%$.

Also, some beam elements, Nos. 37, 48 and 53, for instance, have 'positive' sensitivity, Table 2. For the constraint (1), the most sensitive results are obtained around node 29. The standard deviations of displacement sensitivity with respect to the beam cross-sectional areas are about $15-25 \%$ of the expectations, and stochastic and deterministic results differ by about $3-5 \%$.

The results of displacement sensitivity for the shell elements are less than for the truss and beam elements. Some shell elements, Nos. 18, 38, 48 and 68, for example, are characterized by the positive sensitivity values. The standard deviations of displacement sensitivity with respect to the shell thickness is about $5-20 \%$ of the expectations. Stochastic and deterministic results differ by about $1-3 \%$.

\section{Concluding remarks}

Clearly, sensitivity of stochastic systems is an important topic in the structural analysis, as it allows us to find out the appropriate design point, taking into account random variations in design parameters that reflect the real structural response. Exemplary static sensitivity analysis gives the answer how the design variables affect the response of the system. Apart from the static response and sensitivity results obtained in the deterministic context, the probabilistic setting gives significant information at an uncertain level, i.e., supplying additional results-inaccuracy quality of the obtained solutions.

With the same two probabilistic moment on input, in the suggested perturbation scheme, both the two probabilistic moments are obtained on output, and not the second-order accurate expectations and first-order accurate cross-covariances given by traditional perturbation schemes. The improvement is a few percent, though.

A formulation presented in this paper requires the double summation and, consequently, $o\left(R^{2}\right)$ matrix operations. It is advisable to reduce these operations to $o(R)$ operations by transforming the set of correlated random variables to a set of uncorrelated random variables through a standard eigenproblem, cf. [17]. This approach and a stochastic formulation of dynamic sensitivity are the object of the forthcoming paper.

Open Access This article is distributed under the terms of the Creative Commons Attribution License which permits any use, distribution, and reproduction in any medium, provided the original author(s) and the source are credited.

\section{References}

1. Zienkiewicz, O.C., Taylor, R.L.: The finite element method, vol. 1. McGrew-Hill, London (1989)

2. Zienkiewicz, O.C., Taylor, R.L.: The finite element method, vol. 2. McGrew-Hill, London (1993)

3. Bathe, K.-J.: Finite Element Procedures. Prentice-Hall, New Jersey (1996)

4. Haugh, E.J., Choi, K.K., Komkov, V.: Design Sensitivity Analysis of Structural Systems. Academic Press, Orlando (1986)

5. Mroz, Z., Haftka, R.T.: First- and second-order sensitivity analysis of linear and nonlinear systems. AIAA J. 24, 1187-1192 (1986)

6. Choi, K.K., Kim, N.-H.: Structural Sensitivity Analysis and Optimization. Springer, New York (2010)

7. Drewko, J., Hien, T.D.: First- and second-order sensitivities of beams with respect to cross-sectional cracks. Arch. Appl. Mech. 74, 309-324 (2005) 
8. Ding, J., Pan, Z., Chen, L.: Parameter identification of multibody systems based on second order sensitivity analysis. Int. J. Non-Linear Mech. 47, 1105-1110 (2012)

9. Mroz, Z., Bojczuk, D.: Shape and topology sensitivity analysis and its application to structural design. Arch. Appl. Mech. 82, 1541-1555 (2012)

10. Bojczuk, D., Mroz, Z.: Topological sensitivity derivative with respect to area, shape and orientation of an elliptic hole in a plate. Struct. Multidisc. Optim. 45, 153-169 (2012)

11. Ghanem, R.G., Spanos, P.D.: Stochastic Finite Elements: A Spectral Approach. Springer, New York (1991)

12. Hisada, T., Nakagiri, S.: Stochastic finite element method for structural safety and reliability. Proc. 3rd Int. Conf. Struct. Safety Reliab., 395-402 (1981)

13. Liu, W.K., Belytschko, T., Mani, A.: Random field finite elements. Int. J. Numer. Methods Eng. 23, 1831-1845 (1986)

14. Li, J., Chen, J.: Stochastic Dynamics of Structures. Wiley, Singapore (2009)

15. Greene, M.S., Liu, Y., Chen, W., Liu, W.K.: Computational uncertainty analysis in multiresolution materials via stochastic constitutive theory. Comput. Methods Appl. Mech. Eng. 200, 309-325 (2011)

16. Kleiber, M., Hien, T.D.: The Stochastic Finite Element Method. Wiley, New Jersey (1992)

17. Kleiber, M., Hien, T.D.: Stochastic structural design sensitivity of static response. Int. J. Comput. Struct. 38, 659-667, (1991) Pergamon Press

18. Sluzalec, A.: Stochastic shape sensitivity in powder metallurgy processing. Appl. Math. Model. 36, 3743-3752 (2012)

19. Rahman, S.: Stochastic sensitivity analysis by dimensional decomposition and score functions. Probabilistic Engineering Mechanics 24, 278-287 (2009)

20. Liu, W.K., Belytschko, T., Mani, A.: Probabilistic finite elements for nonlinear structural dynamics. Comput. Methods Appl. Mech. Engrg. 56, 64-81 (1986)

21. Jablonka, A., Hien, T.D.: Damping and dynamic sensitivity (in Polish: Tlumienie a wrazliwosc dynamiczna). Pomiary Automatyka Kontrola 57, 1040-1043 (2011)

22. Jablonka, A.: Numerical dynamic analysis of complex structures (in Polish: Numeryczna analiza dynamiczna konstrukcji zlozonych). Wybrane zagadnienia z dziedziny budownictwa, 535-543, Wyd. Pol. Sl. (2011)

23. Hien, T.D., Kleiber, M.: POLSAP-A Finite Element Code for Deterministic and Stochastic Analyses of Large 3D Structures. IPPT PAN, Warszawa (1990) 